\title{
Global Transcriptional Analysis Reveals Unique and Shared Responses in Arabidopsis thaliana Exposed to Combined Drought and Pathogen Stress
}

\author{
Aarti Gupta, Ananda K. Sarkar and Muthappa Senthil-Kumar * \\ National Institute of Plant Genome Research, New Delhi, India
}

With frequent fluctuations in global climate, plants are exposed to co-occurring drought and pathogen infection and this combination adversely affects plant survival. In the past, some studies indicated that morpho-physiological responses of plants to the combined stress are different from the individual stressed plants. However, interaction

OPEN ACCESS

Edited by:

Mohammad Anwar Hossain, Bangladesh Agricultural University,

Bangladesh

Reviewed by:

Biswapriya Biswavas Misra,

University of Florida, USA

Manoj-Kumar Arthikala

Universidad Nacional Autónoma de

México, Mexico

*Correspondence:

Muthappa Senthil-Kumar skmuthappa@nipgr.ac.in

Specialty section:

This article was submitted to

Plant Biotechnology,

a section of the journal

Frontiers in Plant Science

Received: 11 February 2016

Accepted: 04 May 2016

Published: 24 May 2016

Citation:

Gupta A, Sarkar AK and Senthil-Kumar M (2016) Global Transcriptional Analysis Reveals Unique and Shared Responses in Arabidopsis thaliana Exposed to Combined Drought and Pathogen Stress. Front. Plant Sci. 7:686. doi: 10.3389/fpls.2016.00686 of drought stressed plants with pathogen has not been widely studied at molecular level. Such studies are important to understand the defense pathways that operate as part of combined stress tolerance mechanism. In this study, Arabidopsis thaliana was exposed to individual drought stress, Pseudomonas syringae pv tomato DC3000 (Pst DC3000) infection and their combination. Using Affymetrix WT gene 1.0 ST array, global transcriptome profiling of leaves under individual drought stress and pathogen infection was compared with their combination. The results obtained from pathway mapping (KAAS and MAPMAN) demonstrated the modulation in defense pathways in A. thaliana under drought and host pathogen Pst DC3000 infection. Further, our study revealed "tailored" responses under combined stress and the time of occurrence of each stress during their concurrence has shown differences in transcriptome profile. Our results from microarray and RT-qPCR revealed regulation of 20 novel genes uniquely during the stress interaction. This study indicates that plants exposed to concurrent drought and pathogen stress experience a new state of stress. Thus, under frequently changing climatic conditions, time of occurrence of each stress in the interaction defines the plant responses and should thus be studied explicitly.

Keywords: combined stress, Pseudomonas syringae, microarray, unique pathways, multiple stress tolerance, drought

\section{INTRODUCTION}

Under field conditions drought stress most often occur in conjunction with pathogen infection and this combination negatively impact plant growth (McElrone et al., 2001; Mohr and Cahill, 2003; Choi et al., 2013). Drought potentially alters the plant-pathogen interaction by interfering with the host plant physiological and biochemical processes and making it either more, or less susceptible (Mohr and Cahill, 2003). Alternatively, drought-induced changes directly influence the pathogen 
survival in plant interface making it either more or less virulent (Wiese et al., 2004; Achuo et al., 2006; Goel et al., 2008; Hanso and Drenkhan, 2009; Luck et al., 2011). Furthermore, the combined effect of drought stress and pathogen infection leads to altered resistance responses (Xu et al., 2008; Choi et al., 2013; Prasch and Sonnewald, 2013). Previously, a few studies showed that stress interaction provoke altogether different transcriptome changes that were not seen under either of the individual stresses (Atkinson et al., 2013; Prasch and Sonnewald, 2013). Plant responses to combined drought and pathogen stress has been shown to vary depending on the severity and duration of each stress and also differs with the nature of infecting pathogens (Olson et al., 1990; McElrone and Forseth, 2004; Achuo et al., 2006; Xu et al., 2008). Till date, transcriptome changes in plants under combined drought and pathogen were documented in two studies (Choi et al., 2013; Prasch and Sonnewald, 2013). In the study on Arabidopsis thaliana subjected to drought and Turnip mosaic virus (TuMV) infection, transcriptome profiling revealed the existence of $24 \%$ of the shared genes between individual and combined stress while more than $50 \%$ of the unique genes were noted upon combined stress treatment (Prasch and Sonnewald, 2013). In another study, drought stressed Vitis vinifera showed enhanced disease symptoms under Xylella fastidiosa infection (Choi et al., 2013). Noteworthy, some of the tracheids infecting pathogens have been shown to cause physiological drought (McElrone et al., 2001, 2003). Hence, $X$. fastidiosa and drought stress have exerted synergistic impact on plant-water relations, and under such interaction $56 \%$ of differentially regulated genes were shared with either of the individual stresses (Choi et al., 2013). These studies indicate that the extent of shared responses between combined and individual stresses depends on the nature of pathogens that infect drought stressed plants. However, transcriptome changes in plants under drought and foliar pathogen has not been studied. Such studies are important to understand the genes and pathways that operate uniquely as part of combined stress tolerance mechanism. For plant-bacterial pathogen interaction studies in A. thaliana, Pseudomonas syringae is the best suitable system owing to its resource availability and well established methods (Katagiri et al., 2002). Both water deficit and foliar bacterial pathogen infection have been shown to modulate common plant responses. For example, drought induces stomata closure in the plants (Wilkinson and Davies, 2002), while some foliar bacterial pathogens force open the stomata (Melotto et al., 2008). Such changes thus can impact both this pathogen entry and regulate drought tolerance. In order to understand the modulation of defense reactions in $A$. thaliana under drought and $P$. syringae pv. tomato DC3000 infection, global transcriptome profiling of leaves under individual drought stress, pathogen infection and their combination was performed in this study. Transcriptome profile under different combined stresses was variably influenced by the time of occurrence of each stress during their concurrence. Our study also revealed unique responses under combined stress. Results from this study indicate that plants exposed to combined drought and pathogen stress experience a new state of stress that is different from plants exposed to the individual stresses. Thus, our transcriptomic study indicates the essential role of many unique genes under combined stress and further the existence of common genes between individual and combined stresses suggests a complex crosstalk between stress responsive mechanisms.

\section{MATERIALS AND METHODS}

\section{Plant Material, Bacterial Strain, and Growth Conditions}

Arabidopsis thaliana ecotype Columbia-0 (Arabidopsis Biological Resource Center, accession number CS70000) was used in the study. Seeds were sown in agropeat (Prakruthi Agro Tech, Karnataka, India) and vermiculite (Keltech Energies Ltd, Maharashtra, India) mix (3:1 vol/vol, pre-weighed) and were stratified (for $48 \mathrm{~h}$ in dark at $4^{\circ} \mathrm{C}$ ). Plants were grown under short-day conditions ( $8 \mathrm{~h}$ of light, $16 \mathrm{~h}$ of dark) with $200 \mu \mathrm{E} \mathrm{m} \mathrm{m}^{-2} \mathrm{~s}^{-1}$ light intensity, $75 \%$ humidity, and $20^{\circ} \mathrm{C}$ constant temperature in growth chamber (PGR15, Conviron, Winnipeg, Canada). Plants were bottom irrigated with water or with Hoagland solution (Cat \# TS1094, Himedia Laboratories, Mumbai, India) every alternate day till the start of the stress treatments.

Pseudomonas syringae pv. tomato DC3000 (Pst DC3000), a host pathogen of $A$. thaliana was used in this study. Pst DC3000 was grown in King's B medium (King et al., 1954) supplemented with rifampicin $(50 \mu \mathrm{g} / \mathrm{mL})$, at $28^{\circ} \mathrm{C}$ with continuous shaking of 200 rotations per minute $(\mathrm{rpm})$ for $12 \mathrm{~h}$.

\section{Preparation of Pst DC3000 Inoculum}

Bacterial culture at initial optical density at $600 \mathrm{~nm}\left(\mathrm{OD}_{600}\right)=0.4$ was centrifuged at $4270 \mathrm{~g}$ for $10 \mathrm{~min}$. Bacterial pellet was washed thrice using sterile water and suspended in sterile water to the concentration of $5 \times 10^{3}$ colony forming units $(\mathrm{CFU}) / \mathrm{mL}$.

\section{Combined Stress Imposition}

Drought stress was initiated by withholding water from potted A. thaliana (32-days-old, 8 leaf stage). Gravimetric method (Ramegowda et al., 2013) was followed for stress imposition. Briefly, potted plants were weighed twice a day (11:00 a.m. and 5:00 p.m.) and were brought down to a defined level of drought stress, $40 \%$ soil field capacity $(\mathrm{FC})\left(\Psi_{\mathrm{w}}=-3.9 \mathrm{MPa}\right)$. Control plants were maintained at $100 \%$ FC. According to previous standardizations, the potting mix used in the study attained 40\% FC in 5 days (Supplementary Figure S1A). FC was determined using the following formula;

$$
\mathrm{FC}(\%)=[(\mathrm{WW}-\mathrm{DW}) / \mathrm{DW}] \times 100
$$

WW-wet soil weight; DW-air dry soil weight.

Upon the arrival of $40 \% \mathrm{FC}$ (37-days-old plants), pathogen was infiltrated at $5 \times 10^{3} \mathrm{CFU} / \mathrm{mL}$ concentration through abaxial side of the leaves (DP). The time of pathogen inoculation was considered as $0 \mathrm{~h}$ post-treatment (hpt), such that plant experiences combined drought stress of $40 \%$ FC along with pathogen stress for $24 \mathrm{hpt}$. Individual drought stressed and pathogen infected plants were separately maintained. The control healthy plants were infiltrated only with sterile water (mock inoculation). 
For pathogen first and concurrent drought later treatment, pathogen was infiltrated into the leaves (of 32-days-old-plants) and such plants were subjected to water withdrawal (PD). Individual plants maintained only at $40 \% \mathrm{FC}$ and others treated only with pathogen were also maintained. The outline for combined stress protocol is provided in Supplementary Figure S1A.

\section{Assessment of In Planta Bacterial Multiplication}

Bacterial multiplication in leaves from combined stressed and pathogen only treated plants was assessed at $24 \mathrm{hpt}$. Circular discs measuring $1 \mathrm{~cm}$ diameter were cut out using cork borer from infected leaf. The leaf disc was surface sterilized with $0.01 \% \mathrm{H}_{2} \mathrm{O}_{2}$ for $20 \mathrm{~s}$, and was homogenized in $1000 \mu \mathrm{L}$ of sterile water. Upon further serial dilution in sterile water, it was plated on King's B agar medium supplemented with rifampicin antibiotic $(50 \mathrm{mg} / \mathrm{L})$. Bacterial population was calculated as $\mathrm{CFU} / \mathrm{cm}^{2}$ (Wang et al., 2007). Bacterial numbers were calculated as per the following formula:

Bacterial multiplication $\left(\mathrm{CFU} / \mathrm{cm}^{2}\right)=$

Number of colonies x volume of homogenate $(\mu \mathrm{L})$

$\mathrm{x}$ dilution factor

volume plated

Leaf area $\left(\mathrm{cm}^{2}\right)$

\section{Assessment of Membrane Leakage}

Electrolyte leakage was measured in leaf samples following protocol described by Tripathy et al. (2000). Briefly, discs $(1 \mathrm{~cm}$ diameter) were punched from each leaf and were rinsed in deionized water for $2 \mathrm{~min}$ to remove lysed contents due to cut ends. Washed leaf discs (two discs) were placed in $20 \mathrm{~mL}$ of deionized water for $12 \mathrm{~h}$ with continuous shaking at $60 \mathrm{rpm}$ at $20^{\circ} \mathrm{C}$. Six biological replicates were considered for each treatment. Conductivity of the bathing solution was measured for each sample using conductivity meter (Model-1602, ECTDS-SAL Meter, Esico International, Himachal Pradesh, India). Samples with bathing solution were autoclaved to cause complete (100\%) electrolyte leakage and conductivity was measured again. Electrolyte leakage was expressed as the percentage ratio of initial and final readings.

\section{RNA Extraction}

Leaf tissue (100 mg fresh weight) from third tier of stressed and unstressed rosette was harvested at $24 \mathrm{hpt}$ and immediately frozen in liquid nitrogen. The frozen material was pulverized in liquid nitrogen into a fine powder. The homogenate was used for isolation of total RNA using RNeasy plant mini kit (Cat \# 74904, Qiagen, Hilden, Germany) as per the manufacturer's instructions. RNA samples were treated with DNase I (Cat \# 79254 RNeasy/QlAamp columns, Qiagen, Hilden, Germany) to remove DNA according to the manufacturer's instructions. RNA quality and quantity were evaluated using an Agilent 2100 Bioanalyzer (Agilent Technologies, California, USA) with RNA 6000 Nano Chips (Cat \# 5067-1511, Agilent Technologies,
California, USA), following the manufacturer's protocol. RNA integrity numbers (RIN) ranged from 7.0 to 7.5.

\section{Microarray Hybridization}

Microarray experiment was conducted using Whole Transcript (WT) Expression Arrays (Affymetrix, California, USA). Total RNA was labeled using GeneChip ${ }^{\circledR}$ WT PLUS Reagent Kit (Cat \# 902281, Affymetrix, California, USA) as per manufacturer's protocol. Briefly, total RNA (500 ng) was reverse transcribed to synthesize single-stranded cDNA with T7 promoter sequence at the $5^{\prime}$ end. Template cDNA was converted to doublestranded cDNA, simultaneously degrading the residual RNA. Complimentary RNA (cRNA) was synthesized and amplified by in-vitro transcription of the second-stranded cDNA template using T7 RNA polymerase. cRNA was purified and reverse transcribed to make sense strand cDNA containing dUTP at a fixed ratio relative to dTTP. Template RNA was removed following hydrolysis using RNase $\mathrm{H}$. The purified, sensestrand cDNA is fragmented at the dUTP residues and was labeled using Affymetrix proprietary DNA labeling reagent that is covalently linked to biotin. The appropriate amount of each fragmented and biotin-labeled single stranded (ss)cDNA was mixed with hybridization master mix and was loaded onto cartridge (GeneChip ${ }^{\circledR}$ Gene 1.0 ST, Cat \# 901915, Affymetrix, California, USA). Loaded cartridge was hybridized for $16 \mathrm{~h}$ at $45^{\circ} \mathrm{C}$ and $60 \mathrm{rpm}$ followed by washing, staining, and scanning using GeneChip ${ }^{\circledR}$ hybridization, wash, and stain kit (Cat \# 900720, Affymetrix, California, USA) according to the manufacturer's guidelines. Six different plants were maintained for each treatment and a pool of three plants were sampled for each biological replicate. For each treatment, two biological replicates were hybridized (Supplementary Figure S2A).

\section{Microarray Data Extraction and Analysis}

A concise protocol depicting data analysis is presented in Supplementary Figure S2A. In short, image files (.CEL) were imported into GeneSpring GX 12.1.6 (Agilent Technologies, California, USA). The microarray data was normalized using RMA algorithm (GeneSpring GX 12.1.6). Unpaired $t$-test was employed to obtain differentially expressed genes. More than 2-fold differentially expressed genes (DEGs), between two conditions (treatment over control) with $t$-test $p \leq 0.05$ were selected for further analysis. The selected DEGs were segregated into up- and down-regulated genes. Up-regulated genes were assigned positive values, and down-regulated ones as negative values.

Genes were distributed along gene ontology (GO) biological classes using inbuilt feature of GeneSpring (Agilent GeneSpring GX12.1.6) and relative enrichment was performed as percentage of significantly regulated genes from a specific functional group relative to the total genes from that specific group to the entire chip. Genes were also categorized as per GO molecular function according to TAIR GO functional classification (TAIR 10). DEGs across individual and combined stress treatments were compared using Venn diagrams (Agilent GeneSpring GX12.1.6). Based on Venn intersections, DEGs were segregated as unique 
to combined stress transcriptome and as common between individual and combined stressed transcriptome.

The pathway networks were determined by input of the selected gene lists into KEGG Automatic Annotation Server (KAAS, http://www.genome.jp/tools/kaas/) and MAPMAN (http://mapman.gabipd.org/web/guest/mapman). Heat maps were drawn with fold change values using GENE-E software (http://www.broadinstitute.org/cancer/software/GENE-E/).

\section{Quantitative Real-Time PCR Analysis}

Transcript expression of selected genes was quantified by realtime PCR (RT-qPCR) using ABI Prism 7000 sequence detection system (Applied Biosystems, California, USA). For all the RT-qPCR experiments, three independent biological replicates were performed. Total RNA $(5 \mu \mathrm{g})$ was reverse transcribed to make template (first strand $\mathrm{cDNA}$ ) in a reaction volume of $50 \mu \mathrm{L}$ using verso cDNA synthesis kit (Cat \# AB1453A, Thermo Scientific, Massachusetts, USA). Gene-specific primers (Supplementary Table S1) were designed using Primer 3 software (Untergrasser et al., 2012). Template cDNA was diluted (5fold) and was mixed with $750 \mathrm{nM}$ each of the specific primers and SYBR Green PCR master mix (Applied Biosystems, USA) in a final volume of $10 \mu \mathrm{L}$. Ct values obtained for AtACTIN2 (AT3G18780) gene was used to normalize data. Fold change in gene expression in stressed samples was quantified using comparative D cycle threshold (CT) method relative to the nonstressed control samples (Livak and Schmittgen, 2001).

\section{Statistical Analysis}

Data presented are average of biological replicates. The numbers of biological replicates considered for each experiment are mentioned in the legend for respective figure. Error bars represent \pm SEM. Significant differences among treatments were determined by one-way ANOVA and applying the leastsignificant difference post-hoc Tukey's test $(p<0.05)$ (SigmaPlot 11.0, Systat Software Inc., California, USA). The test of significance applied in RT-qPCR analysis was Student's $t$-test $(p<0.05)$ (SigmaPlot 11.0, Systat Software Inc., California, USA).

\section{RESULTS}

\section{Combined Stress Imposition Lead to Drought and Pathogen Defined Plant Physio-Morphological Changes}

Leaves of Arabidopsis thaliana were exposed to combined drought and bacterial pathogen (Pst DC3000) stress by following two different protocols. In the first protocol, drought stress was started first and then pathogen inoculation was done (hereafter referred as DP). Initially, plants were maintained at $100 \%$ field capacity (FC), which corresponds to $\sim 92 \%$ leaf relative water content (RWC) and at the start of the experiment, water was withheld so that pots come down to $40 \%$ soil moisture content (FC) with leaves exhibiting 55\% RWC (Supplementary Figure S1). In the DP protocol, plants were allowed to experience gradual drought and the pathogen was inoculated at $40 \%$ field capacity (FC). Plants were maintained at this drought level along with continued in vivo pathogen multiplication for $24 \mathrm{~h}$. Gravimetric assessment revealed that plants realized progressive drought at 80 and 60\% FC for 1 and 2 days respectively before reaching to a final drought stress level of 40\% FC (Supplementary Figure S1A). Individual stressed plants namely drought plants at $40 \% \mathrm{FC}$ and pathogen inoculated plants along with absolute control and mock (water only) inoculated plants were maintained as controls and data gathered were compared with appropriate controls (Supplementary Figure S1A).

In the second protocol, pathogen was inoculated and then the irrigation was stopped to induce drought stress. In planta bacterial numbers at $0 \mathrm{hpt}$ reflect delivery of $2.14 \mathrm{Log}\left(\mathrm{CFU} / \mathrm{cm}^{2}\right)$ concentration of pathogen in the inoculated leaf. Drought stress at $40 \%$ FC had reached 5 days after pathogen inoculation. During this period, both exponential pathogen multiplication and progressive drought stress were observed in these plants (Supplementary Figure S1). At 40\% FC with progressive pathogen multiplication, plants were maintained for $24 \mathrm{~h}$ and samples were then taken for experiments. This protocol where pathogen was inoculated before start of drought stress is referred as PD. Individual stressed plants namely, drought stressed and pathogen infiltrated (for 6 days of prolonged duration, referred as PP) along with mock inoculated and absolute control plants were maintained in this experiment. Another control involving mock (water) infiltration into the leaves of drought stressed plants (DM) was also included for transcriptomic study. Raw and processed result files generated from microarray experiment were submitted to GEO NCBI (accession no. GSE79681). Results on RWC, in planta bacterial numbers and transcriptomic analysis from combined stressed plants indicated successful drought stress imposition and pathogen infection (Supplementary Figures S1C,D, S3). Results from transcriptome analysis revealed presence of drought and pathogen responsive genes examples, those encoding for late embryogenesis abundant proteins (LEAs), dehydrins, chitinases and pathogenesis related (PR) proteins (Welin et al., 1994; Katagiri et al., 2002; Zhao et al., 2003; Hanin et al., 2011; Yang et al., 2012), among the most differentially expressed transcripts in response to stress (Supplementary Figure S3). Further, the results on gene ontology biological process revealed that the top-most differentially expressed genes (DEGs) were enriched with GO biological process "response to stress" (Supplementary Figure S4).

Influence of combined stress on pathogen infection and disease progression was studied and we observed reduced in planta bacterial multiplication in DP stressed plants. However, PD stressed plants showed bacterial numbers similar to pathogen only inoculated plants. Accordingly, DP stressed plants did not show disease-induced chlorosis, but conspicuous chlorosis was observed in PD stressed plants (Supplementary Figure S1B). Also, the RWC in DP and PD stressed plants was 55 and $46 \%$ leaf water content respectively and no significant difference in RWC was found between DP and PD. Since independently occurring drought and pathogen induces leakage of solutes through cellular membranes, electrical conductivity measurement was considered as parameter for assessing stress impact in combined stressed plants. Membrane leakage was higher under combined DP stress $(38 \%)$ in comparison to the drought $(26 \%)$ or pathogen $(22 \%)$ 
stresses. However, in case of the combined PD stress (33\%), the leakage was at par with pathogen (PP) induced damage (34\%) (Supplementary Figure S1E). All these results indirectly suggest that pathogen infection does not alter the drought stress specific impact on plants in both the combined stress protocols. In this manuscript, the changes in plant defense against pathogen influenced by drought stress are explored.

Our experiments revealed two major inferences. One, the first occurring individual stress likely plays role in deciding outcome of stress interaction. Based on the initial analysis of transcriptome data (Supplementary Figures 1A,B) we predicted existence of both unique and shared responses that can explain the observed physio-morphological changes. Second, the likely "dominant" stressor, drought, seem to play role in reducing the bacterial multiplication in DP plants, but not in PD plants. The transcriptomic data for DP and individual stress controls revealed changes in plant defense responses. Hence, we first present the analysis of the net effect of combined stresses (DP and PD) on plants. In second part of the manuscript, we present transcriptome analysis and provide reasons for reduction in bacterial numbers in DP or pathogen number in PD plants similar to pathogen infiltrated plants.

\section{Combined Stressed Plants Showed Distinct Transcriptome Changes}

In order to understand the transcriptome changes in combined stressed plants, whole-transcripts expression arrays were performed using RNA samples isolated from plants (leaf tissue) exposed to individual and combined stress conditions (Supplementary Figure S2A). Hierarchical clustering based on expression values of the transcripts showed that the two replicates of differentially treated plants clustered together (Supplementary Figure S2B). Drought stressed plant infiltrated with water (drought-mock, DM) clustered closely to the drought stressed plants, inferring that the syringe infiltration method (water and wound response) used in this study are accommodated in our analysis so that the exclusive treatment effects are compared. Both the combined stress treatments (DP and PD) were close to the drought stress clade whereas transcriptome profile under pathogen and prolonged pathogen treatment formed a separate clade, possibly reflecting the dominance of drought stressor in the combined stress (Supplementary Figure S2B).

We present DP and PD transcriptome changes under two major categories of responses. One is exclusive to combined stress, i.e., "unique" and the second encompasses common genes between individual and combined stresses that either have expression pattern similar to individual stress termed as "shared" or those with "tailored" expression pattern compared to individual stresses. Unpaired $t$-test listed out the significant and differentially regulated genes in each stress condition compared with respective controls. Transcriptome profile under each condition is listed in Supplementary File S1. Figures 1A,B illustrates the numbers of statistically significant differentially expressed transcripts under each stress condition (File S1). For instance, the number of differentially regulated transcripts in individual drought and pathogen stress and their combination were 655, 558, and 834 respectively. However, when pathogen was infiltrated ahead of drought stress (PD) imposition, there was a pronounced increase in the number of differentially regulated transcripts as 1647 and 1716 under prolonged pathogen (PP) and PD combined stress respectively.

Venn intersections depicted the unique and common transcriptional responses (or common) between individual and combined stress. Comparison of differentially expressed genes across individual and combined stress treatments revealed 37 genes common among drought, pathogen and DP treatments and 104 genes common among drought, prolonged pathogen and PD treatments. These common genes however showed shared or tailored expression between individual and combined stress. As presented in heat maps, 19 genes were shared and 18 genes were tailored in DP stress compared to the individual stresses (Figure 1C). Similarly 59 genes showed shared expression pattern among individual drought, prolonged pathogen and PD combined stress while, 45 genes showed tailored expression pattern between individual and combined stressed plants (Figure 1D). Our analysis revealed a substantial number of genes which were induced exclusively upon combined stress treatment (unique genes), were absent from the list of DEGs under individual drought or pathogen treatments. Combined DP stressed plants exhibited 505 unique DEGs and combined PD plants exhibited 885 numbers of unique DEGs. Strikingly, when the unique transcripts under DP stress were compared with that of unique $\mathrm{PD}$, a large number of genes were found to be common between the two. Moreover, expression pattern of these genes under DP and PD stress was also similar (Supplementary Figure S5). Comparative expression profile of "stress category" genes from the list of total DEGs under combined DP and PD stress revealed $\sim 35 \%$ of common stress genes. Moreover these common stress genes exhibited similar expression pattern under DP and PD stress (Supplementary Figure S8). This indicates that, in order to combat combined stress (involving drought and bacterial pathogen), possibly plants tend to modulate a certain number of basal combined stress responsive genes.

Few of the unique transcripts under DP stress (in comparison to $\mathrm{D}$ and $\mathrm{P}$ stress, based on gene IDs) belong to a gene family. The others members of such gene families were observed to be differentially expressed under individual drought or pathogen stress (File S2). The expression pattern of the different isoforms of a gene may depend on the nature of the stress. These included calcium-dependent lipid-binding domain-containing protein, leucine rich repeat (LRR) transmembrane protein, transcriptions factors from NAC, WRKY, and MYB families. The membrane localized LRR proteins have been implicated in sensing the external environment while members of these transcription factor families are involved in signal transduction and plant responses to various abiotic and biotic stresses (Jones and Dangl, 2006; Zheng et al., 2007; Dubos et al., 2010; Nuruzzaman et al., 2013; Osakabe et al., 2013; Bakshi and Oelmüller, 2014; Nakashima et al., 2014).

To identify the key genes involved in combined stress responses, we analyzed expression pattern of 20 genes unique to combined DP stress through RT-qPCR. Few of these 
A

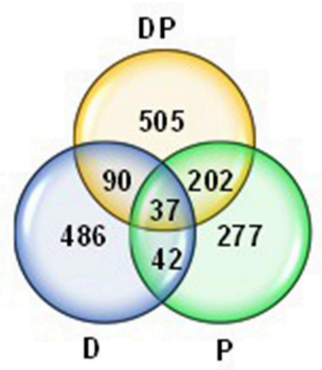

C

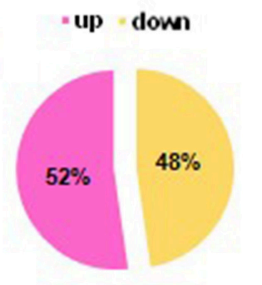

G

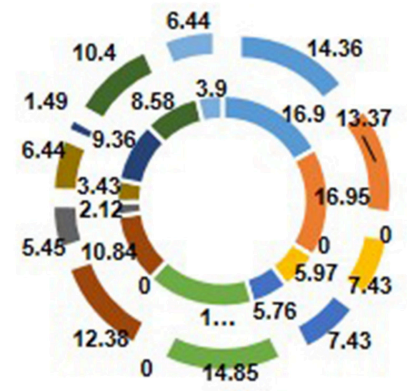

- cellular process

- metabolic process

- reproductive process

- cellular component organizationbiogenesis

- protein sorting

- regulation of growth

B

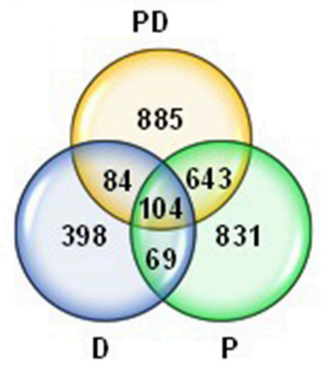

D up down

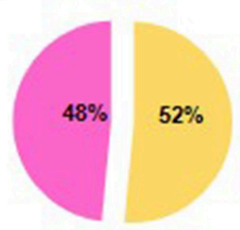

H

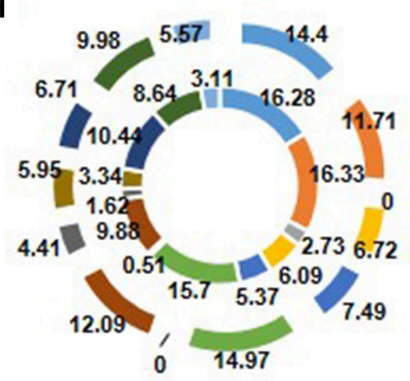

- regulation of circadian rythm

- response to stimulus

- immune system process

- signaling

- tissue/organ developmental process

- unknown biological process

- others

$\mathbf{J}$

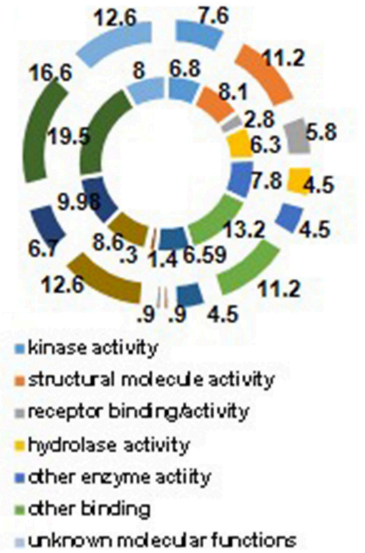

tos
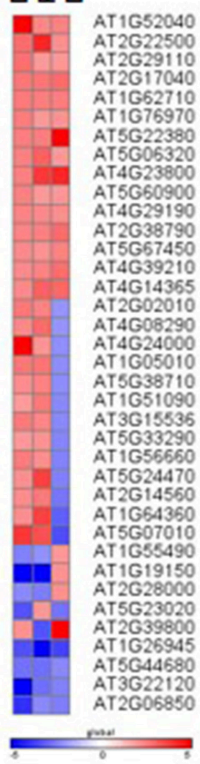

F

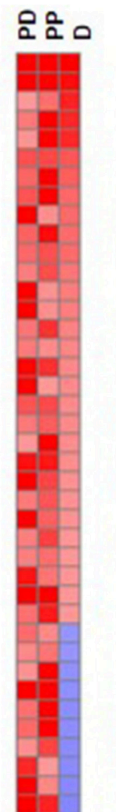

ATSO222380

AT4012735

AT 4023800

AT3 13080

AT2624600

ATSG 15500

AT2G17040

AT2 238790

AT1673540

AT1G67920

ATSG67450

AT 345290

AT2G28400

AT3647090

AT4G37640

AT2G26560

AT2G250

AT2G35070

AT4G34390

AT1970

ATA 323180

ATSG60900

AT2029110

ATSG06320

AT2002010

AT4027830

AT4032940

AT 4024000

ATSO38710

AT1662300

AT1 1662300
ATS 513330

AT3G15536

AT20330\%

AT107792

AT3C51430

ATSG3329

AT1613830

ATs

AT1602470

AT1G27020

AT4G33040

ATSG22300

AT2G14560

AT4G1937

AT3659700

AT2G43000

ATS 1970

AT1G47890

ATT 164360

AT1G69310

AT3G 10320

AT2G25440

AT261461

AT3 3222235

AT2 241190

ATSG63530

ATSG 2440

ATSG23020

AT2041940

AT1662380

ATSG57760

AT2028000

AT1619770

AT3052720

AT34

AT 4034260

AT160220

AT 4024770

ATSG47540

AT3605020

ATSG67335

AT3G59940

AT3G11210

ATCGO0460

AT1662480

AT3G22120

AT3654920

ATSG44680

AT3654400

AT4G31590

AT4G01080

AT4G10120

AT2 36830

AT 3 G 24480

AT3G22142

AT1626945

AT2606850

AT1674670

AT1619150

AT3022120

FIGURE 1 | Summary of comparative transcriptome profile of combined stressed plants with individual stressed plants. Arabidopsis thaliana plants (32-days-old) were exposed to individual stresses, i.e., drought (D), Pseudomonas syringae pv. tomato DC3000 (Pst DC3000; P). Combined stress treatments involved superimposition of pathogen infection on drought stressed plants (DP) or drought imposition in already pathogen infected plants (PD). Microarray experiment was carried out using Affymetrix WT gene chip array and differentially expressed genes (DEGs) in each stress treatment were identified in comparison to control 
FIGURE 1 | Continued

samples (fold change $>2$, unpaired $t$-test $p<0.05$ ). Venn intersection between DEGs in individual and combined stress highlights the unique and shared transcriptome profile in response to DP and PD combined stress (A,B). Percent distribution of uniquely expressed genes in up- and down-regulated category in response to combined DP and PD stress treatments is shown here (C,D). Expression pattern of genes shared among D, P, and DP; D, PP, and PD is depicted as heat map (E,F). Color bar scale shows the fold change range with red and blue color representing up- and down-regulation respectively. Gene ontology (GO) biological function based functional categorization (Agilent GeneSpring GX12.1.6) was done for DEGs unique to combined stress and shared between D, P and DP (G) D, PP and PD (H). GO molecular function based categorization (as per TAIR) was done for DEGs unique to combined stress and shared between D, P and DP (I) and D, PP and PD (J). Outer ring represents the GO categories for genes shared between individual and combined stress treatments. Inner ring represents the genes unique to the combined stress treatments. Gene names and descriptions for the gene IDs presented in the figure are provided in Supplementary File S2.

candidate genes belong to multigene family (other isoforms are implicated in individual stress tolerance, File S2). These genes include those encoding for ring finger protein, receptor like kinase, plant natriuretic peptide (PNP), sugar transporter, sodium hydrogen exchanger and nuclear transcription factor. For example, earlier literature indicated that PNPs are secreted into the apoplast and elicit a range of host defense responses such as tissue specific modifications of cation transport, changes in stomatal conductance and the photosynthetic rate (Turek et al., 2014). RT-qPCR results showed expression pattern similar to microarray for the tested genes indicating induction of these genes under combined stress (Figure 2; Supplementary Figure S7). Gene encoding ABA insensitive ring protein 2, a RING/Ubox superfamily protein was up-regulated under both drought and pathogen stress but was induced to 15-folds upon combined DP stress. This gene has previously been shown to be involved in drought stress and pathogen infection (Fabro et al., 2008; Cho et al., 2011). Another uniquely expressed transcription factor was NAC with transmembrane motif1 (NTM1). As reflected in our RT-qPCR results the expression pattern of this gene was unique to combined stress and presence of transcripts under drought or pathogen only stress was observed only to the minimal levels. This is a membrane protein tethered to ER or nuclear membrane and possibly has a role in ER stress, like other members of this family (Kørner et al., 2015). Stay green 2 encoding a chlorophyll catabolic protein showed up-regulation under pathogen stress but under combined stress it was induced to 23-fold change. This could be plant's counter defense to pathogen induced chlorophyll degradation. Our result is further strengthened by the information curated from public (eFP browser) and literature data under drought stress or Pst DC3000 infection. The genes showing unaltered expression under drought or Pst DC3000 infection are more suitable candidates to be established as "unique genes" under combined stress and are likely to be involved in plant adaptations to combined stress (over individual stress).

\section{Unique and Shared Genes Were Specifically Enriched for Pathways Contributing to Defense and Growth}

Genes unique to the combined stress and genes common between individual and combined stress treatments were further classified to inferred biological process using the Gene Ontology (GO) terminologies provided by GeneSpring. Our results implicate that GO terms were similarly enriched with unique genes from DP and PD combined stress treatments (Figures 1E-I,
Supplementary Figure S6; Supplementary Tables S2, S3). Likewise common genes were similarly enriched with GO terms under both kinds of combined stress treatments (Figures 1E-I; Supplementary Tables S2, S3). However, a comparison of unique vs. common genes hinted toward prioritized plant responses under combined stress for growth, signaling and immune system responses. For instance, the GO term "growth" was completely absent from the common genes and at the same time "signaling" term was less enriched with common genes in comparison to the unique genes under DP stress (Supplementary Table S3). Unique genes induced under DP and PD combined stress were associated with similar pathways (mapped through KAAS) viz., carbon, nitrogen, sulfur, fatty acid and amino acid metabolism, photosynthesis, secondary metabolites and wax biosynthesis. In spite of the common stressors involved, the two kinds of combined stresses, i.e., DP and PD invoked different pathways (Supplementary Tables S4, S5). For example, unique DEGs under DP stress mapped to inositol phosphate metabolism, thiamine metabolism, folate biosynthesis and $\mathrm{ABC}$ transporter while PD unique genes mapped to linoleic acid metabolism, glycan degradation and nucleic acid synthesis, repair, transport and degradation (Supplementary Tables S4, S5). DP combined stress activated diterpenoid biosynthesis while monoterpenoid biosynthesis was induced upon PD combined stress (Supplementary Tables S4, S5). Thus, the two different protocols followed for combined stress actually represented two different kinds of combined stress, possibly the time of stress decides the plant defense responses.

\section{Basal Disease Resistance Pathway Might Contribute to Drought-Induced Endurance to Pathogen Infection}

In order to delineate the drought induced changes in plant defenses, the DEGs under drought stress, pathogen infection and combined DP stress were surveyed through MAPMAN and KAAS. Host plant defenses against pathogen characterized by PAMP triggered immunity (PTI) or effector triggered immunity (ETI) and virulence factors were analyzed (Figure 3A). The comparative transcriptome analysis under individual and DP stress shows that the expression pattern of these defense genes under combined stress could not be delineated from either of the individual stresses. These observations suggest that plants elicit different isoforms of a defense gene under individual and combined stresses and possibly opt different route to combat them (Figure 3B). Our results show that the genes involved in 

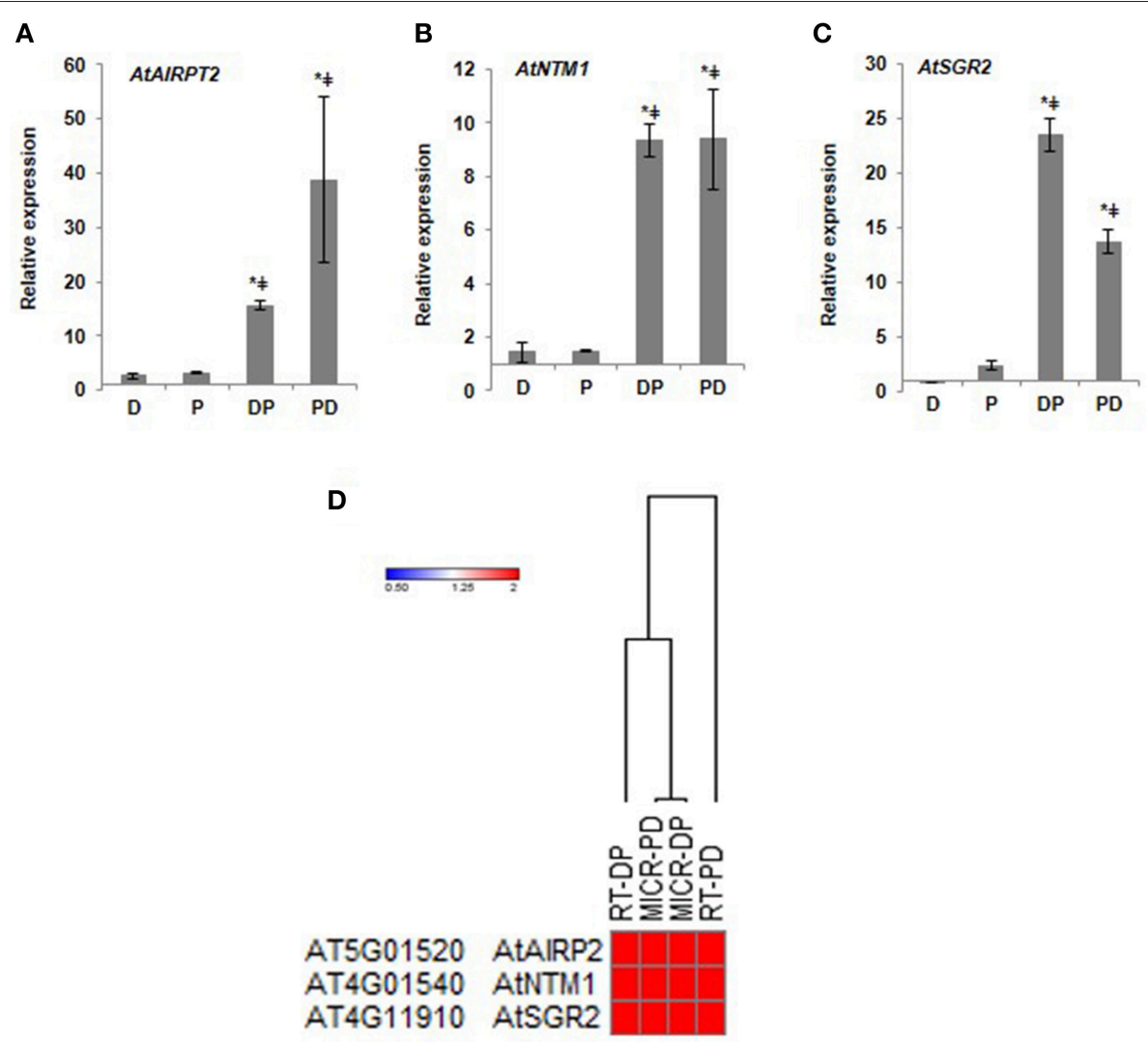

FIGURE 2 | RT-qPCR validation of microarray data from DP combined stressed leaves. Relative expression of AtAIRP2 (A) AtNTM1 (B), AtSGR2 (C) genes is presented. Candidate genes were selected and corresponding transcript accumulation under combined stress treatment was quantified by RT-qPCR. Fold change in expression levels relative to the control samples were normalized to AtACTIN2 gene expression. Each experiment was carried out with three biological and two technical replicates. Data represents the average of three biological replicates and error bars shows standard error of mean (SEM). Significance was calculated using Student's $t$-test where * and $\ddagger$ symbol shows significance at $p<0.05$ over drought and pathogen stress respectively. Heat map depicts the correlation between microarray and qRT-PCR data. Dendrogram was based on Euclidean distance (D). MICR, fold change values from microarray experiment; RT, fold change values from RT-qPCR analysis. Color bar in red and blue color represents up- and down-regulated genes respectively. Gene names and descriptions for the gene IDs presented in the figure are provided in Supplementary File S2.

PTI were induced upon DP stress where genes involved in MAP kinase signaling were specifically induced in response to bacterial PAMP, flagellin (FLG) recognition, for example, flagellin-sensing 2 (FLS) receptor (Figure 3B). The FLS mediated signaling was however suppressed in pathogen only infected plants. The defense related genes like AtFRK1 and $P R$ were highly induced in response to DP stress while compared to pathogen infection (Figure 3B). ETI related genes however did not show much variation in response to DP stress over pathogen infection. Genes influenced by coronatine, a bacterial virulence factor showed variation in expression pattern. Genes encoding JAZ protein were specifically influenced by different individual and combined stress treatments. AtJAZ3 and AtJAZ10 were up-regulated under DP stress, while these were down-regulated under pathogen infection. Apart from PTI, ETI and virulence factor influenced genes, there are certain plant genes which are direct targets of pathogen effectors and contribute to pathogen virulence. These genes were either up-regulated or uninfluenced under drought stress but were mostly down-regulated under pathogen infection
(Figure 3B). Comparison with DP stress indicated up-regulation of these genes (Figure 3B).

\section{Priming with Host Pathogen Suppressed Some Plant Defense Responses during Combined Stress}

Nitric oxide-associated protein 1 (NOA1) is involved in nitric oxide biosynthesis and is implicated in various abiotic stresses and pathogen triggered susceptibility (PTS) (Asai and Yoshioka, 2008). The expression of AtNOA1 was reduced in PD stress (Supplementary Figure S9A). AtCYP79B3 gene encoding a tryptophan $\mathrm{N}$-hydroxylase is induced by PAMP (Geng et al., 2012) and is involved in the production of precursor of tryptophan-derived metabolites viz., camalexin and glucosinolates (Hull et al., 2000; Gigolashvili et al., 2007). Expression pattern of this gene was also down-regulated in PD stress (Supplementary Figure S9A). Expression pattern of defense genes including TIR/TIR-NBS-LRR class or defensin gene was down-regulated in PD combined stress (Supplementary 
A

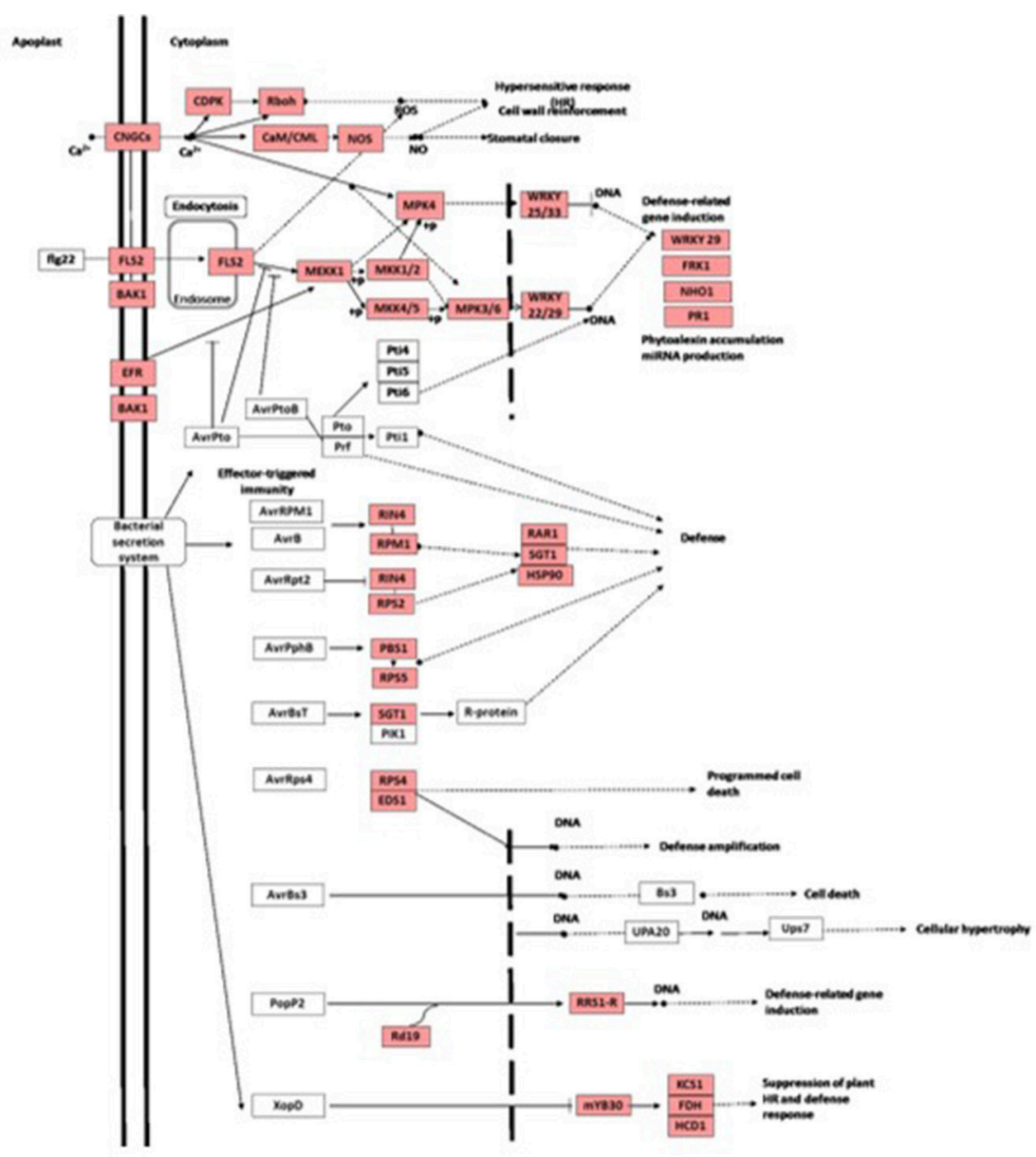

B

PTI

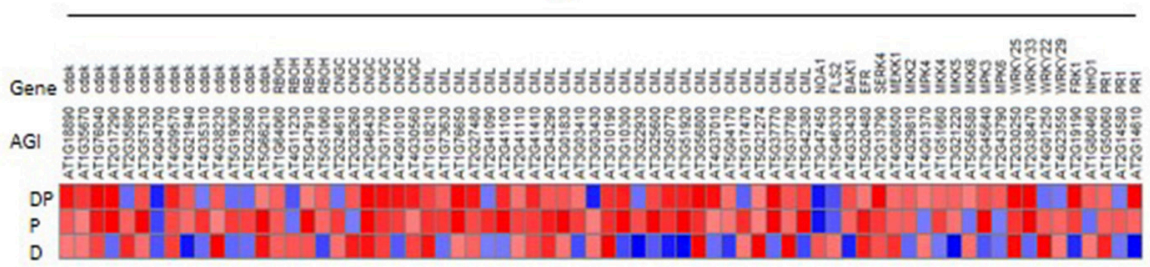

ETI

COR
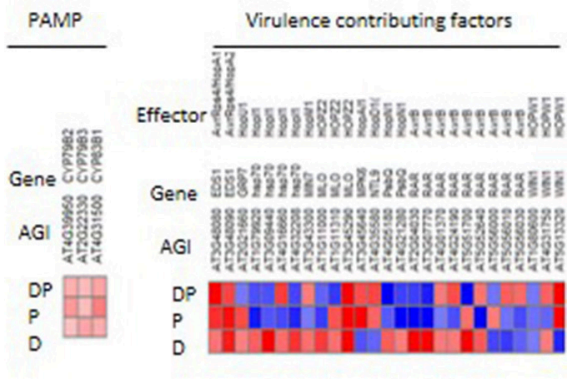

FIGURE 3 | Modulation in expression pattern of "defense" related transcripts under combined DP stress. Illustration depicts bacterial PAMP and effector triggered pathways during plant-pathogen interaction (redrawn from KEGG) (A). Heat map represents expression pattern of different genes involved in plant-pathogen interaction (B). Differentially expressed genes were listed out using Affymetrix ${ }^{\circledR}$ Expression Console (EC) and Transcriptome Analysis Console (TAC). DEGs under DP combined stress were associated with PAMP triggered immunity (PTI) and effector triggered immunity (ETI), according to annotation provided by KAAS. Genes influenced by bacterial virulence factor coronatine (COR), PAMP or different bacterial effectors (secreted into the plant cell and contributing to the virulence) were manually curated. Fold change values were used to plot heat map where color bar scale shows the range with red and blue color representing up- and down-regulation respectively. AGI, A. thaliana gene IDs provided by TAIR. Gene IDs and gene names with descriptions are provided in Supplementary File S2. 
Figure S9A). Expression of a gene encoding coronatine target protein JAZ10 was also down-regulated under PD stress (Supplementary Figure S9A). Down-regulation of defense genes in PD transcriptome hints toward suppression of some host defenses upon priming with pathogen in combined stress. Thus, although the transcriptome profiles under DP and PD stress were highly correlated, differential expression of the few genes (like those discussed in this section) could possibly underlie the reason for loss of drought induced tolerance under PD stress.

\section{Drought Induced Changes in Defense Related Pathways during Combined Stress}

Our analysis revealed up-regulation of genes involved in proline degradation in response to pathogen stress and up-regulation of AtP5CS1 and down-regulation of AtProDH2 under drought stress (Supplementary Figure S9B). The observed trend is in line with the involvement of $\mathrm{P} 5 \mathrm{C}$ in pathogen defense responses (Qamar et al., 2015) and accumulation of proline under drought stress (Sharma et al., 2011). However, under combined DP stress we observed up-regulation of both proline biosynthesis and proline degradation. This hints toward the maintenance of proline homeostasis under DP stress which seems to be important while sustaining plant growth under stressed condition (Kavi Kishor and Sreenivasulu, 2014).

Likewise, expression profile of polyamine metabolism genes revealed up-regulation of genes involved in polyamine biosynthesis during individual drought and pathogen stresses, but combined stressed plants exhibited up-regulation of both biosynthesis as well as catabolism related genes (Supplementary Figure S9C). Similar observations were made by Hatmi et al. (2014). This points toward the maintenance of polyamine homeostasis under combined stress in order to keep up with the tradeoff between growth and combined stress responses.

The results on expression profile of proline and polyamine metabolism genes thus indicate that the combined stressed plants are preferentially protected against the adverse effects of drought and pathogen induced damages.

\section{DISCUSSION}

Arabidopsis interaction with Pst DC3000 is well studied (Katagiri et al., 2002; Nobuta and Meyers, 2005; Thilmony et al., 2006). Alteration in plant water relations and the modes of physiological responses to reduce the pathogen multiplication in the intracellular spaces has been demonstrated (Freeman and Beattie, 2009; Beattie, 2011). However, the molecular events involved in its interaction in the presence of drought are not known. Importantly, understanding the changes provoked by plants at the interface of simultaneous drought (Osakabe et al., 2014) and pathogen stress is important to know the "net impact" of combined stresses (Ramegowda and Senthil-Kumar, 2015). In this study we showed that the transcriptome profile of $A$. thaliana differs in response to DP and PD combined stresses. We first illustrated the expression of unique batch of genes to combined stress, apart from common genes' expression that are consistent with either of individual stresses studied here. In the later, importantly, we show that a batch of common genes' expression are "tailored" in response to combined stress and their expression pattern is not seen in either of individual stresses. This indicated that plants likely perceive combined stresses as new form of stress, different from individual stresses. One of the previous studies (Choi et al., 2013) on Vitis vinifera interaction with simultaneous drought and $X$. fastidiosa, also showed large number of common genes with differential expression pattern and some genes unique to combined stress, indicating that plant uses best possible existing stress responsive molecular machinery for upcoming new or additional stresses, which is rather an economic strategy.

\section{DP Combined Stress Imposition Reduced In Planta Bacterial Multiplication}

Our study showed that transcriptome changes in drought stressed plants reduced the subsequent pathogen multiplication in DP (over individual pathogen stress), not in PD plants, indicating the time of occurrence of first stress is important determinant of the combined stress interaction with plants (Supplementary Figures S1B,D). We hypothesized that the reduction in pathogen multiplication could be due to change in water relation status of plant physiologically not supporting the pathogen or/and priming for robust defense. Freeman and Beattie (2009) in support of former notion, indicated that localized cessation of water availability in the pathogen infected plants by restricted vascular flow coupled with stomatal loss can reduce bacterial number. Although this was in respect of Pst DC3000 expressing avrRpm1 that provoke gene-for-gene mediated defense responses, the early induction of the localized water limitation in infected tissues could be attributed to basal and/or PAMP-mediated responses. Nevertheless, in our study delivery of pathogen in water by syringe inoculation and only moderate reduction in RWC values of drought stressed plants at the time of pathogen inoculation suggest that water limitation could not be major reason for reduced number of pathogens at 24 hpt (Supplementary Figure S1C). Even the moderate increase in membrane leakage (Supplementary Figure S1E) that could have released more nutrients from the cell in to the apoplast did not facilitate increased multiplication, indicating the possibility of robust defense in the drought stressed plants (DP). Hence, we attribute the later notion, contribution by cell mounted defense, for the reduction in bacterial number as observed in plants responding to drought stress (Osakabe et al., 2014) in our study. In order to explore the details of plant-pathogen interaction under DP, we divided these plant defense responses into three categories namely, non-specific inducible chemical and structural changes, PTI and ETI-mediated changes. Pathogen counter defenses, including virulence factors and ETS are also considered.

\section{Differential Induction of Robust Defenses Likely Lead to Reduced Bacterial Number in DP Stressed Plants}

Under DP stress, the plant's unique responses consisted of genes involved in diterpenoid biosynthesis, $\mathrm{ABC}$ transporter and inositol phosphate metabolism pathways that contribute to 
inducible immune responses like callose deposition, cell wall thickening, phenyl propanoids and other secondary metabolite production. Unique genes expressed under combined stresses were specifically enriched with GO biological process "growth." GO term "response to stress" was prevalent in both unique as well as common genes. These results implies an impact on growth processes while negotiating with the basal stress responses, under combined interaction of two different stressors. Furthermore, expression pattern of stress related genes under DP stress was consistent with drought only gene expression data which indicate that such defense responses are primed in the DP interaction.

Apoplast located pathogen is known to inject effector proteins through type-3 secretion system (Katagiri et al., 2002) and secrete virulence factors (Bender et al., 1999), like coronatine into plant cell to favor its multiplication. Coronatine (COR), JA mimic produced by Pst DC3000 is known to promote this pathogen multiplication and chlorosis by suppressing the SA-mediated defense pathways (Melotto et al., 2008). It is likely that DP combined stressed plants did not suppress SA-pathway as suggested by expression of SA signaling related genes (Supplementary Figure S10). Further, JA related gene expression was high in DP (Supplementary Figure S10). These two evidences can be used to speculate that either the DP plants did not facilitate COR synthesis in pathogen (or its delivery in the plants) or the COR could not facilitate suppression of SA pathway genes which could have been overwhelmed by the SA-JA-ABA hormonal signaling primed by drought stress. Since, the virulence of Pst DC3000 is independent of COR, or at the least only has quantitative effect, we looked at the relevance of effector proteins-their cognate plant receptor genes through DP transcriptome.

Contrary to DP observation, HopAM1 effector had been shown to increase the virulence of an otherwise weak pathogen under drought stress in Wassilewskija (Ws-0) accession of A. thaliana (Goel et al., 2008). Our DP microarray profile showed only marginal manipulation of ABA responses (Supplementary Figure S10) that are reported in that study that likely lead to suppression of defense responses in Ws plants. Based on our data, this can be explained by following two ways. First, the Ws-0 is known to have endogenously high and high drought induced ABA levels (North et al., 2007), that suppress several defense responses (Anderson et al., 2004; Fan et al., 2009), compared to Col-0 plants. Second, unlike weak Pma M6C $\Delta \mathrm{E}$ ( $P$. syringae $\mathrm{pv}$. maculicola) pathogen strains used in that study, HopAM1 is disposable in Pst DC3000 and virulence was not changed in HopAM1 mutant pathogens. Hence, HopAM1 less likely played a role in combined stress in Col-0. Nevertheless, our observations does not dispel the notion that the diverse effectors produced by Pst DC3000 could exploit the host machinery under drought and favor high infection of a weak pathogen. It is logical to infer that less virulent pathogens could have increased pathogenesis (Kazan and Lyons, 2014), especially in PD like combined stresses. Contrary to the DP, high bacterial multiplication in the PD combined stressed plants indicate that the established pathogen infection before drought stress possibly suppress the plant defense responses.

Further, one of the study on Verticillium interaction with A. thaliana showed that this pathogen infection enhances drought tolerance (Reusche et al., 2012). Both in DP and PD, we did not find physiological evidences to suggest alteration in the drought specific effects. However, we have observed several changes in the genes attributed to be involved in drought tolerance under stress combination. At this point, we do not have evidences to comment on its impact on either the "net combined stress effect" or the drought-like effect in the combined stressed plants.

\section{CONCLUSIONS}

In this manuscript we not only profiled the entire transcriptome changes in combined stressed plants and dissected the unique and shared responses, but also provided brief explanation for the reduced pathogen multiplication, as observed in other plant species, based on the changes in gene regulation. It is likely that both the priming of basal defenses and modulation due to interaction of drought specific and pathogen derived responses in DP plants contributed for this reduction. We also infer that the timing of drought stress decides the outcome of plant interaction with the pathogen infection. Further systematic functional validation studies are needed to elucidate the specific genes involved in the response of DP and PD combined stressed plants.

\section{AUTHOR CONTRIBUTIONS}

MS conceived the idea. MS and AG designed the study. AG performed the experiments. AG analyzed the data with the input from AS. MS and AG wrote the manuscript. AS, AG, and MS critically read the manuscript.

\section{ACKNOWLEDGMENTS}

Projects at MS lab are supported by National Institute of Plant Genome Research core funding and Department of Biotechnology (DBT) DBT-Ramalingaswami re-entry fellowship grant (BT/RLF/re-entry/23/2012). We also thank Mr. Sundar and Mr. Prem Negi for extending technical help at the laboratory and central instrumentation facility respectively.

\section{SUPPLEMENTARY MATERIAL}

The Supplementary Material for this article can be found online at: http://journal.frontiersin.org/article/10.3389/fpls.2016. 00686 


\section{REFERENCES}

Achuo, E. A., Prinsen, E., and Höfte, M. (2006). Influence of drought, salt stress and abscisic acid on the resistance of tomato to Botrytis cinerea and Oidium neolycopersici. Plant Pathol. 55, 178-186. doi: 10.1111/j.13653059.2006.01340.x

Anderson, J. P., Badruzsaufari, E., Schenk, P. M., Manners, J. M., Desmond, O. J., Ehlert, C., et al. (2004). Antagonistic interaction between abscisic acid and jasmonate-ethylene signaling pathways modulates defense gene expression and disease resistance in Arabidopsis. Plant Cell 16, 3460-3479. doi: $10.1105 /$ tpc. 104.025833

Asai, S., and Yoshioka, H. (2008). The role of radical burst via MAPK signaling in plant immunity. Plant Signal. Behav. 3, 920-922. doi: 10.4161/psb.6601

Atkinson, N. J., Lilley, C. J., and Urwin, P. E. (2013). Identification of genes involved in the response of Arabidopsis to simultaneous biotic and abiotic stresses. Plant Physiol. 162, 2028-2041. doi: 10.1104/pp.113.222372

Bakshi, M., and Oelmüller, R. (2014). WRKY transcription factors jack of many trades in plants. Plant Signal. Behav. 9:e27700. doi: 10.4161/psb.27700

Beattie, G. A. (2011). Water relations in the interaction of foliar bacterial pathogens with plants. Ann. Rev. Phytopathol. 49, 533-555. doi: 10.1146/annurev-phyto073009-114436

Bender, C. L., Alarcón-Chaidez, F., and Gross, D. C. (1999). Pseudomonas syringae Phytotoxins: mode of action, regulation, and biosynthesis by peptide and polyketide synthetases. Microbiol. Mol. Biol. Rev. 63, 266-292.

Cho, S. K., Ryu, M. Y., Seo, D. H., Kang, B. G., and Kim, W. T. (2011). The Arabidopsis RING E3 ubiquitin ligase AtAIRP2 plays combinatory roles with AtAIRP1 in abscisic acid-mediated drought stress responses. Plant Physiol. 157, 2240-2257. doi: 10.1104/pp.111.185595

Choi, H.-K., Iandolino, A., Goes da Silva, F., and Cook, D. (2013). Water deficit modulates the response of Vitis vinifera to the Pierce's disease pathogen Xylella fastidiosa. Mol. Plant Microbe Interact. 26, 643-657. doi: 10.1094/MPMI-09-120217-R

Dubos, C., Stracke, R., Grotewold, E., Weisshaar, B., Martin, C., and Lepiniec, L. (2010). MYB transcription factors in Arabidopsis. Trends Plant Sci. 15, 573-581. doi: 10.1016/j.tplants.2010.06.005

Fabro, G., Di Rienzo, J. A., Voigt, C. A., Savchenko, T., Dehesh, K., Somerville, S., et al. (2008). Genome-wide expression profiling Arabidopsis at the stage of Golovinomyces cichoracearum haustorium formation. Plant Physiol. 146, 1421-1439. doi: 10.1104/pp.107.111286

Fan, J., Hill, L., Crooks, C., Doerner, P., and Lamb, C. (2009). Abscisic acid has a key role in modulating diverse plant-pathogen interactions. Plant Physiol. 150, 1750-1761. doi: 10.1104/pp.109.137943

Freeman, B. C., and Beattie, G. A. (2009). Bacterial growth restriction during host resistance to Pseudomonas syringae is associated with leaf water loss and localized cessation of vascular activity in Arabidopsis thaliana. Mol. Plant Microbe Interact. 22, 857-867. doi: 10.1094/MPMI-22-7-0857

Geng, X., Cheng, J., Gangadharan, A., and Mackeya, D. (2012). The coronatine toxin of Pseudomonas syringae is a multifunctional suppressor of Arabidopsis defense. Plant Cell 24, 4763-4774. doi: 10.1105/tpc.112.105312

Gigolashvili, T., Yatusevich, R., Berger, B., Müller, C., and Flügge, U. I. (2007). The R2R3-MYB transcription factor HAG1/MYB28 is a regulator of methioninederived glucosinolate biosynthesis in Arabidopsis thaliana. Plant J. 51, 247-261. doi: 10.1111/j.1365-313X.2007.03133.x

Goel, A. K., Lundberg, D., Torres, M. A., Matthews, R., Akimoto-Tomiyama, C., Farmer, L., et al. (2008). The Pseudomonas syringae type III effector HopAM1 enhances virulence on water stressed plants. Mol. Plant Microbe Interact. 21, 361-370. doi: 10.1094/MPMI-21-3-0361

Hanin, M., Brini, F., Ebel, C., Toda, Y., Takeda, S., and Masmoudi, K. (2011). Plant dehydrins and stress tolerance versatile proteins for complex mechanisms. Plant Signal. Behav. 6, 1503-1509. doi: 10.4161/psb.6.10.17088

Hanso, M., and Drenkhan, M. (2009). Diplodia pinea is a new pathogen on Austrian pine (Pinus nigra) in Estonia. Plant Pathol. 58, 797. doi: 10.1111/j.1365-3059.2009.02082.x

Hatmi, S., Gruau, C., Trotel-Aziz, P., Villaume, S., Rabenoelina, F., Baillieul, F., et al. (2014). Drought stress tolerance in grapevine involves activation of polyamine oxidation contributing to improved immune response and low susceptibility to Botrytis cinerea. J. Exp. Bot. 66, 775-787. doi: $10.1093 /$ jxb/eru436
Hull, A. K., Vij, R., and Celenza, J. L. (2000). Arabidopsis cytochrome P450s that catalyze the first step of tryptophan-dependent indole-3acetic acid biosynthesis. Proc. Natl. Acad. Sci. U.S.A. 97, 2379-2384. doi: 10.1073/pnas.040569997

Jones, J. D. G., and Dangl, J. L. (2006). The plant immune system. Nature 444, 323-329. doi: $10.1038 /$ nature 05286

Katagiri, F., Thilmony, R., and Heb, S. Y. (2002). The Arabidopsis thalianaPseudomonas syringae interaction. Arabidopsis Book 1:e0039. doi: 10.1199/tab.0039

Kavi Kishor, P. B., and Sreenivasulu, N. (2014). Is proline accumulation per se correlated with stress tolerance or is proline homeostasis a more critical issue? Plant Cell Environ. 37, 300-311. doi: 10.1111/pce.12157

Kazan, K., and Lyons, R. (2014). Intervention of phytohormone pathways by pathogen effectors. Plant Cell 26, 2285-2309. doi: 10.1105/tpc.114.125419

King, E. O., Ward, M. K., and Raney, D. E. (1954). Two simple media for the demonstration of pyocyanin and fluorescein. J. Lab. Clin. Med. 44, 301-307.

Kørner, C. J., Du, X., Vollmer, M. E., and Pajerowska-Mukhtar, K. M. (2015). Endoplasmic reticulum stress signaling in plant immunity-at the crossroad of life and death. Int. J. Mol. Sci. 16, 26582-26598. doi: 10.3390/ijms161125964

Livak, K. J., and Schmittgen, T. D. (2001). Analysis of relative gene expression data using real-time quantitative PCR and the $2^{-\Delta \Delta \mathrm{CT}}$ Method. Methods 25, 402-408. doi: 10.1006/meth.2001.1262

Luck, J., Spackman, M., Freeman, A., Trebicki, P., Griffiths, W., Finlay, K., et al. (2011). Climate change and diseases of food crops. Plant Pathol. 60, 113-121. doi: $10.1111 / j .1365-3059.2010 .02414 . x$

McElrone, A. J., and Forseth, I. N. (2004). Photosynthetic responses of a temperate liana to Xylella fastidiosa infection and water stress. J. Phytopathol. 152, 9-20. doi: 10.1046/j.1439-0434.2003.00794.x

McElrone, A. J., Sherald, J. L., and Forseth, I. N. (2001). Effects of water stress on symptomatology and growth of Parthenocissus quinquefolia infected by Xylella fastidiosa. Plant Dis. 85, 1160-1164. doi: 10.1094/PDIS.2001.85.11.1160

McElrone, A. J., Sherald, J. L., and Forseth, I. N. (2003). Interactive effects of water stress and xylem-limited bacterial infection on the water relations of a host vine. J. Exp. Bot. 54, 419-430. doi: 10.1093/jxb/erg046

Melotto, M., Underwood, W., and He, S. H. (2008). Role of stomata in plant innate immunity and foliar bacterial diseases. Ann. Rev. Phytopathol. 46, 101-122. doi: 10.1146/annurev.phyto.121107.104959

Mohr, P. G., and Cahill, D. M. (2003). Abscisic acid influences the susceptibility of Arabidopsis thaliana to Pseudomonas syringae pv. tomato and Peronospora parasitica. Funct. Plant Biol. 30, 461-469. doi: 10.1071/FP02231

Nakashima, K., Yamaguchi-Shinozaki, K., and Shinozaki, K. (2014). The transcriptional regulatory network in the drought response and its crosstalk in abiotic stress responses including drought, cold, and heat. Front. Plant Sci. 5:170. doi: $10.3389 /$ fpls.2014.00170

Nobuta, K., and Meyers, B. C. (2005). Pseudomonas versus Arabidopsis: models for genomic research into plant disease resistance. BioScience 55, 679-686. doi: 10.1641/0006-3568(2005)055[0679:PVAMFG]2.0.CO;2

North, H. M., De Almeida, A., Boutin, J. P., Frey, A., To, A., Botran, L., et al. (2007). The Arabidopsis ABA-deficient mutant aba4 demonstrates that the major route for stress-induced ABA accumulation is via neoxanthin isomers. Plant J. 50, 810-824. doi: 10.1111/j.1365-313X.2007.03094.x

Nuruzzaman, M., Sharoni, A. M., and Kikuchi, S. (2013). Roles of NAC transcription factors in the regulation of biotic and abiotic stress responses in plants. Front. Microbiol. 4:248. doi: 10.3389/fmicb.2013.00248

Olson, A. J., Pataky, J. K., D’Arcy, C. J., and Ford, R. E. (1990). Effects of drought stress and infection by maize dwarf mosaic virus on sweet corn. Plant Dis. 74, 147-151. doi: 10.1094/PD-74-0147

Osakabe, Y., Osakabe, K., Shinozaki, K., and Tran, L.-S. P. (2014). Response of plants to water stress. Front. Plant Sci. 5:86. doi: 10.3389/fpls.2014. 00086

Osakabe, Y., Yamaguchi-Shinozaki, K., Shinozaki, K., and Tran, L.-S. P. (2013). Sensing the environment: key roles of membrane-localized kinases in plant perception and response to abiotic stress. J. Exp. Bot. 64, 445-458. doi: $10.1093 / \mathrm{jxb} / \mathrm{ers} 354$

Prasch, C. M., and Sonnewald, U. (2013). Simultaneous application of heat, drought, and virus to Arabidopsis plants reveals significant shifts in signaling networks. Plant Physiol. 162, 1849-1866. doi: 10.1104/pp.113.2 21044 
Qamar, A., Mysore, K. S., and Senthil-Kumar, M. (2015). Role of proline and pyrroline-5-carboxylate metabolism in plant defense against invading pathogens. Front. Plant Sci. 6:503. doi: 10.3389/fpls.2015.00503

Ramegowda, V., and Senthil-Kumar, M. (2015). The interactive effects of simultaneous biotic and abiotic stresses on plants: mechanistic understanding from drought and pathogen combination. J. Plant Physiol. 176, 47-54. doi: 10.1016/j.jplph.2014.11.008

Ramegowda, V., Senthil-Kumar, M., Ishiga, Y., Kaundal, A., Udayakumar, M., and Mysore, K. S. (2013). Drought stress acclimation imparts tolerance to Sclerotinia sclerotiorum and Pseudomonas syringae in Nicotiana benthamiana. Int. J. Mol. Sci. 14, 9497-9513. doi: 10.3390/ijms14059497

Reusche, M., Thole, K., Janz, D., Truskina, J., Rindfleisch, S., Drübert, C., et al. (2012). Verticillium infection triggers VASCULAR-RELATED NAC DOMAIN7-dependent de novo xylem formation and enhances drought tolerance in Arabidopsis. Plant Cell. 24, 3823-3837. doi: 10.1105/tpc.112.103374

Sharma, S., Villamor, J. G., and Verslues, P. E. (2011). Essential role of tissuespecific proline synthesis and catabolism in growth and redox balance at low water potential. Plant Physiol. 157, 292-304. doi: 10.1104/pp.111.183210

Thilmony, R., Underwood, W., and He, S. Y. (2006). Genome-wide transcriptional analysis of the Arabidopsis thaliana interaction with the plant pathogen Pseudomonas syringae pv. tomato DC3000 and the human pathogen Escherichia coli O157:H7. Plant J. 46, 34-53. doi: 10.1111/j.1365-313X.2006.02725.x

Tripathy, J. N., Zhang, J., Robin, S., Nguyen, T. T., and Nguyen, H. T. (2000). QTLs for cell-membrane stability mapped in rice (Oryza sativa L.) under drought stress. Theoret. App. Genet. 100, 1197-1202. doi: 10.1007/s001220051424

Turek, I., Marondedze, C., Wheeler, J. I., Gehring, C., and Irving, H. R. (2014). Plant natriuretic peptides induce proteins diagnostic for an adaptive response to stress. Front. Plant Sci. 5:661. doi: 10.3389/fpls.2014.00661

Untergrasser, A., Cutcutache, I., Koressaar, T., Ye, J., Faircloth, B. C., Remm, M., et al. (2012). Primer3 - new capabilities and interfaces. Nucleic Acids Res. 40:e115. doi: 10.1093/nar/gks596

Wang, D., Pajerowska-Mukhtar, K., Culler, A. H., and Dong, X. (2007). Salicylic acid inhibits pathogen growth in plants through repression of the auxin signaling pathway. Curr. Biol. 17, 1784-1790. doi: 10.1016/j.cub.2007.09.025
Welin, B. V., Olson, A., Nylander, M., and Palva, E. T. (1994). Characterization and differential expression of $\mathrm{dhn} / \mathrm{lea} / \mathrm{rab}$-like genes during cold acclimation and drought stress in Arabidopsis thaliana. Plant Mol. Biol. 26, 131-144. doi: $10.1007 / \mathrm{BF} 00039526$

Wiese, J., Kranz, T., and Schubert, S. (2004). Induction of pathogen resistance in barley by abiotic stress. Plant Biol. 6, 529-536. doi: 10.1055/s-2004-8 21176

Wilkinson, S., and Davies, W. J. (2002). ABA-based chemical signalling: the coordination of responses to stress in plants. Plant Cell Environ. 25, 195-210. doi: 10.1046/j.0016-8025.2001.00824.x

Xu, P., Chen, F., Mannas, J. P., Feldman, T., Sumner, L. W., and Roossinck, M. J. (2008). Virus infection improves drought tolerance. New Phytol. 180, 911-921. doi: 10.1111/j.1469-8137.2008.02627.x

Yang, Y., He, M., Zhu, Z., Li, S., Xu, Y., Zhang, C., et al. (2012). Identification of the dehydrin gene family from grapevine species and analysis of their responsiveness of various forms of abiotic and biotic stress. BMC Plant Biol. 54:140. doi: 10.1186/1471-2229$12-140$

Zhao, Y., Thilmony, R., Bender, C. L., Schaller, A., He, S. Y., and Howe, G. A. (2003). Virulence system of Pseudomonas syringae pv. tomato promote bacterial speck disease in tomato by targeting the jasmonate signaling pathway. Plant J. 36, 485-499. doi: 10.1046/j.1365-313X.2003.01895.x

Zheng, Z., Mosher, S. L., Fan, B., Klessig, D. F., and Chen, Z. (2007). Functional analysis of Arabidopsis WRKY25 transcription factor in plant defense against Pseudomonas syringae. BMC Plant Biol. 7:2. doi: 10.1186/1471-2229-7-2

Conflict of Interest Statement: The authors declare that the research was conducted in the absence of any commercial or financial relationships that could be construed as a potential conflict of interest.

Copyright (c) 2016 Gupta, Sarkar and Senthil-Kumar. This is an open-access article distributed under the terms of the Creative Commons Attribution License (CC BY). The use, distribution or reproduction in other forums is permitted, provided the original author(s) or licensor are credited and that the original publication in this journal is cited, in accordance with accepted academic practice. No use, distribution or reproduction is permitted which does not comply with these terms. 